\title{
Gender perceptions on eating disorders slow to change
}

$\mathrm{C}$

onsider these two words: eating disorder. What image springs to mind? A father of three who works in the oil industry? A male lawyer? A young man with muscular shoulders on a college wrestling team?

Chances are, these were not the people you first associated with an eating disorder. Most likely, you thought of a very different type of person.

"A young adolescent girl from a relatively privileged background," says Leigh Cohn, author of Making Weight: Men's Conflicts with Food, Weight, Shape \& Appearance. "That was the image because that was the type of person showing up for treatment. All the emphasis was on women."

For many years, it was thought that men accounted for only about $10 \%$ of cases of eating disorders such as anorexia nervosa and bulimia. But this estimate, based on people in treatment, is now believed to be way off the mark. A growing body of research suggests as many as $25 \%$ of people with these conditions are male, and for binge eating, that increases to about $40 \%$.

Though a decade's worth of research indicates eating disorders in men are a bigger problem than imagined, the public perception that these are "female diseases" lingers. This certainly isn't helping to get more men to recognize they have a problem and need treatment, experts suggest - especially considering the stigma already associated with eating disorders and the fact that men are less likely to seek medical treatment in general.

"Raising awareness is probably the most important thing. We have to lower the bar a little bit about being concerned when men lose a lot of weight," says Dr. Blake Woodside, medical director of the eating disorders program at the Toronto General Hospital in Ontario and a professor in the psychiatry department at the University of Toronto.

"Parents don't think about this for skinny teenage boys. Boys grow tall quickly and are expected to be skinny for a while," adds Woodside, whose

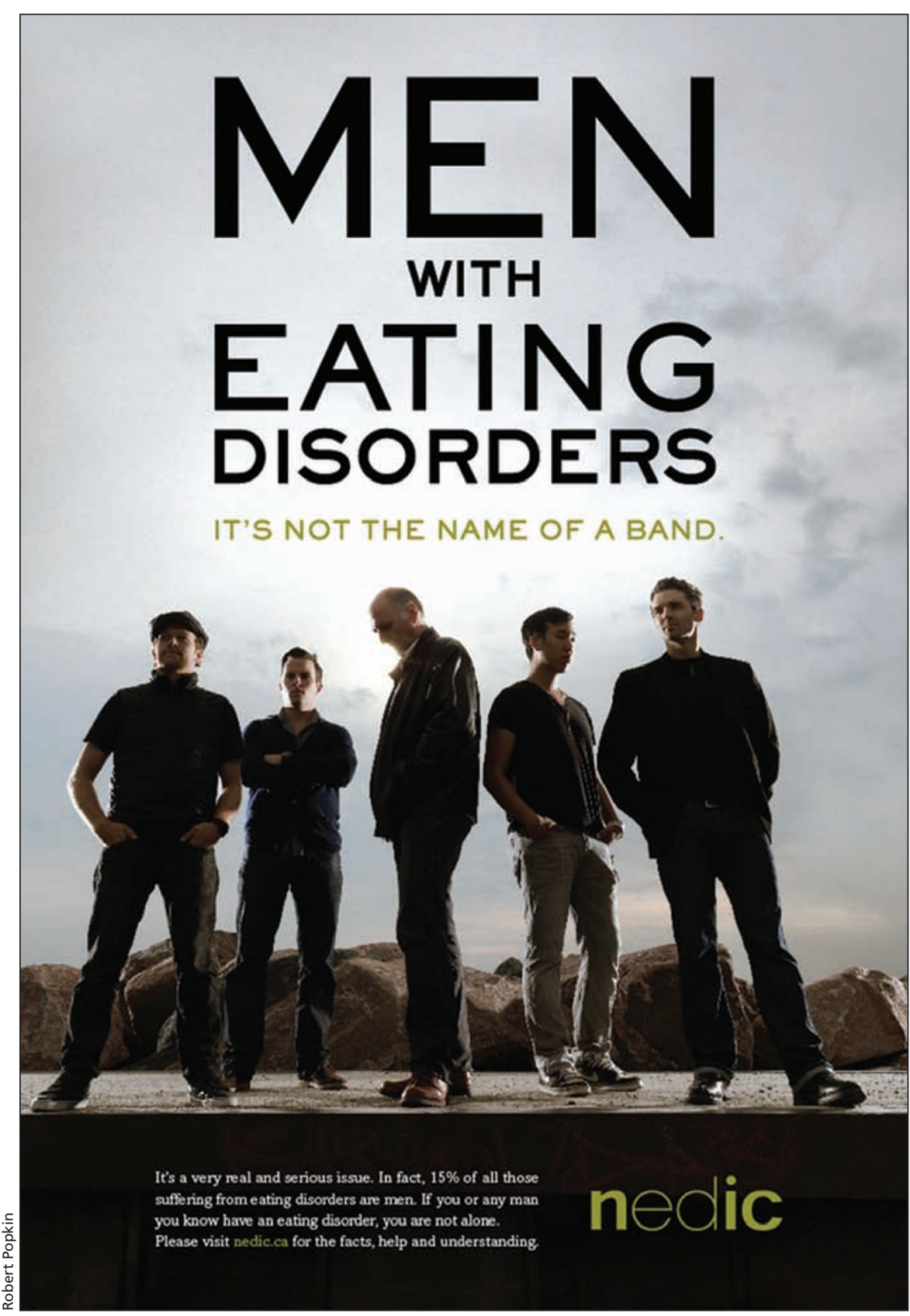

The National Eating Disorder Information Centre recently launched a poster and pamphlet campaign to raise awareness about men with eating disorders.

research indicates men and women who do seek treatment for eating disorders are similar in clinical presentation (Am J Psychiatry 2001;158:570-4).

One difference between genders, however, is that men with eating disorders seem to be more likely to also have other mental health issues, such as anxiety, depression and obsessivecompulsive disorder. A study of male twins in Finland, for instance, concluded that anorexia nervosa "in males in the community is more common, transient and accompanied by more 
substantial comorbidity than previously thought" (PLoS ONE 2009;4:e4402).

"I was surprised by how many problems they had," says lead author Dr. Anu Raevuori, a postdoctoral research fellow in the department of public health at the University of Helsinki in Finland. "They had a pretty rough life."

It was also quite apparent that the stigma of having a disorder typically associated with females made men reluctant to talk about it. "When I interviewed twin males with eating disorders, many of them hadn't even told their co-twin," says Raevuori. "I was the first person they were telling."

The reasons men and women develop eating disorders are similar. It can be about projecting emotional issues onto the body, having a sense of control over food or poor body image. In recent years, the male body has become objectified in popular media just as much as female bodies, says Dr. Ted Weltzin, medical director of eating disorders services at Rogers Memorial Hospital in
Oconomowoc, Wisconsin. As a result, the diet and exercise industry have begun targeting men, offering special fitness and nutrition regimes with promises to make them lean and muscular.

"These things introduced men to dieting in a way that was never seen before," says Weltzin. "It opened up this big diet and weight-loss market for men."

This appears to be an even bigger problem among homosexual men. They live in a community where there is tremendous pressure to look fit, according to David Brennan, assistant professor in the Factor-Inwentash Faculty of Social Work at the University of Toronto. In a study of participants in Pride Toronto 2008, Brennan found that men who have sex with men report high levels of body dissatisfaction and are at higher risk for eating disorders (Int J Mens Health 2011;10:253-68).

"There is a strong focus in the culture on the body and body image," says Brennan. "It is heavily influenced by media, by imagery in gay clubs, gay bars and pornography. It does impact how people feel about their bodies."

Though there is no malicious intent behind the lack of attention given to males with eating disorders, it is high time that the problem be corrected, according to Merryl Bear, director of the National Eating Disorder Information Centre, a nonprofit organization based in Toronto. "It's been a longstanding gap in the areas of understanding early intervention and treatment in eating disorders," she says. "Men have been neglected in that area."

To redress that, her organization recently launched a poster and pamphlet campaign (www.nedic.ca/store /poster_psa5.shtml). Eating disorders can be devastating, Bear says, not only to the body but also the mind. "Malnutrition impacts everything. We are losing some of our brightest minds and most compassionate people." - Roger Collier, CMAJ

CMAJ 2013. DOI:10.1503/cmaj.109-4360 